\title{
Spotlight influenza: Extending influenza surveillance to detect non-influenza respiratory viruses of public health relevance: analysis of surveillance data, Belgium, 2015 to 2019
}

Lorenzo Subissi $^{1,2}$, Nathalie Bossuyt ${ }^{3}$, Marijke Reynders44, Michèle Gérard55, Nicolas Dauby5,6, Patrick Lacor7, Siel Daelemans ${ }^{8}$

, Bénédicte Lissoir ${ }^{2}$, Xavier Holemans ${ }^{10}$, Koen Magerman ${ }^{11,12}$, Door Jouck ${ }^{12}$, Marc Bourgeois ${ }^{13}$, Bénédicte Delaere ${ }^{13}$, Sophie

Quoilin $^{3}$, Steven Van Gucht ${ }^{1}$, Isabelle Thomas ${ }^{1}$, Cyril Barbezange ${ }^{1}$

1. National Influenza Centre, Sciensano, Brussels, Belgium

2. European Public Health Microbiology Training Programme (EUPHEM), European Centre for Disease Prevention and Control, Stockholm, Sweden

3. Epidemiology of Infectious Diseases, Sciensano, Brussels, Belgium

4. Department of Laboratory Medicine, Medical Microbiology, Algemeen Ziekenhuis Sint-Jan, Brugge-Oostende AV, Belgium

5. Centre Hospitalier Universitaire St-Pierre, Brussels, Belgium

6. Centre for Environmental Health and Occupational Health, School of Public Health, Université Libre de Bruxelles (ULB), Brussels, Belgium

7. Internal Medicine-Infectious Diseases, Universitair Ziekenhuis Brussel, Brussels, Belgium

8. Pediatric Pulmonary and Infectious Diseases, Universitair Ziekenhuis Brussel, Brussels, Belgium

9. Microbiology, Grand Hôpital de Charleroi, Charleroi, Belgium

10. Infectiology, Grand Hôpital de Charleroi, Charleroi, Belgium

11. Clinical Laboratory, Jessa Ziekenhuis, Hasselt, Belgium

12. Infection Control, Jessa Ziekenhuis, Hasselt, Belgium

13. Centre Hospitalier Universitaire UCL Namur, Ysoir, Belgium

Correspondence: Cyril Barbezange (cyril.barbezange@sciensano.be)

Citation style for this article:

Subissi Lorenzo, Bossuyt Nathalie, Reynders Marijke, Gérard Michèle, Dauby Nicolas, Lacor Patrick, Daelemans Siel, Lissoir Bénédicte, Holemans Xavier,

Magerman Koen, Jouck Door, Bourgeois Marc, Delaere Bénédicte, Quoilin Sophie, Van Gucht Steven, Thomas Isabelle, Barbezange Cyril. Spotlight influenza:

Extending influenza surveillance to detect non-influenza respiratory viruses of public health relevance: analysis of surveillance data, Belgium, 2015 to 2019 . Euro Surveill. 2021;26(38): pii=2001104. https://doi.org/10.2807/1560-7917.ES.2021.26.38.2001104

Background: Seasonal influenza-like illness (ILI) affects millions of people yearly. Severe acute respiratory infections (SARI), mainly influenza, are a leading cause of hospitalisation and mortality. Increasing evidence indicates that non-influenza respiratory viruses (NIRV) also contribute to the burden of SARI. In Belgium, SARI surveillance by a network of sentinel hospitals has been ongoing since 2011. Aim: We report the results of using in-house multiplex $q P C R$ for the detection of a flexible panel of viruses in respiratory ILI and SARI samples and the estimated incidence rates of SARI associated with each virus. Methods: We defined ILI as an illness with onset of fever and cough or dyspnoea. SARI was defined as an illness requiring hospitalisation with onset of fever and cough or dyspnoea within the previous 10 days. Samples were collected in four winter seasons and tested by multiplex qPCR for influenza virus and NIRV. Using catchment population estimates, we calculated incidence rates of SARI associated with each virus. Results: One third of the SARI cases were positive for NIRV, reaching $49.4 \%$ among children younger than 15 years. In children younger than 5 years, incidence rates of NIRV-associated SARI were twice that of influenza (103.5 vs 57.6/100,000 person-months); co-infections with several NIRV, respiratory syncytial viruses, human metapneumoviruses and picornaviruses contributed most (33.1, 13.6, 15.8 and 18.2/100,000 person-months, respectively). Conclusion: Early testing for NIRV could be beneficial to clinical management of SARI patients, especially in children younger than 5 years, for whom the burden of NIRV-associated disease exceeds that of influenza.

\section{Introduction}

Acute viral infections of the respiratory tract are common in humans. According to the World Health Organization (WHO), complications such as lower respiratory tract infections and pneumonia are among the main causes of mortality in children and elderly people worldwide [1]. The burden attributed to seasonal influenza virus has long received most of the attention [2], but the involvement of non-influenza respiratory viruses (NIRV), such as respiratory syncytial virus and human metapneumovirus, is increasingly documented $[3,4]$. However, the burden of NIRV [5-7] compared with seasonal influenza [8] is not sufficiently estimated [9]. These data would allow to better understand the need for enhanced surveillance of these viruses during winter seasons with the aim of improving patient management. 


\section{FIGURE 1}

Flow diagram for participant inclusion in the study for surveillance of influenza-like illness and severe acute respiratory infections, Belgium, 2015-2019 (n = 9,734)

\section{A. Influenza-like illness}

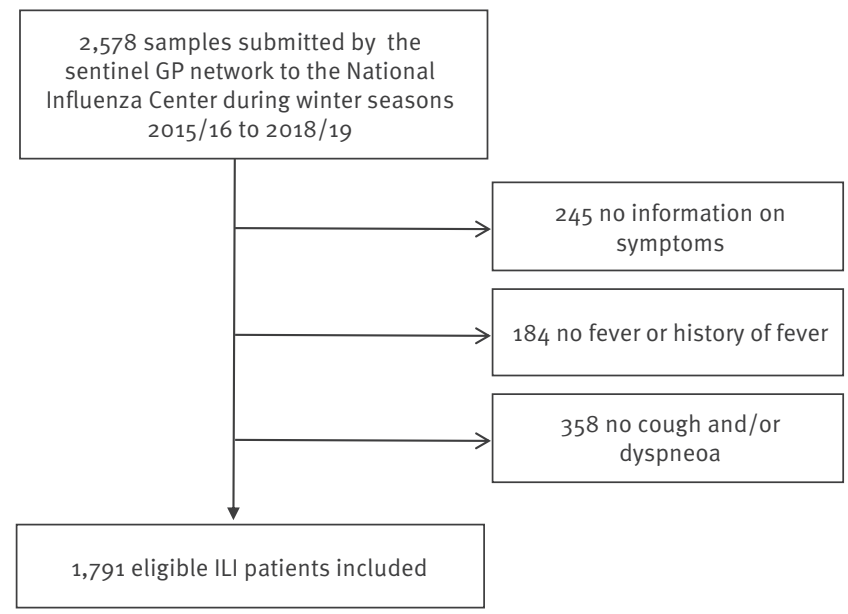

B. Severe acute respiratory infection

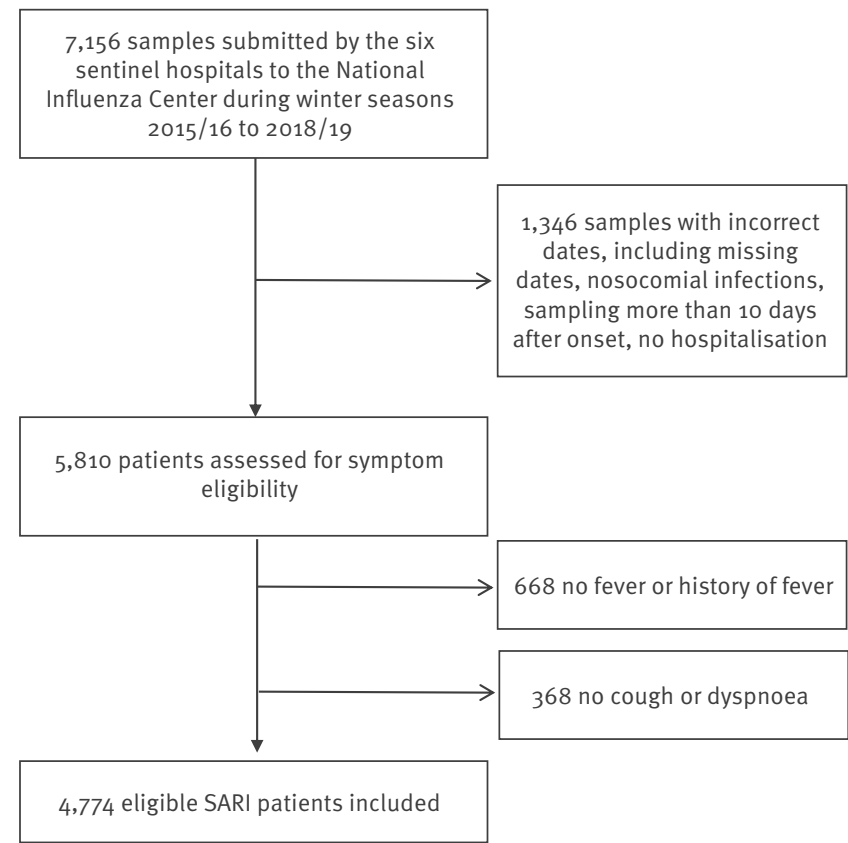

GP: general practitioner; ILI: influenza-like illness; SARI: severe acute respiratory infection.

The WHO implemented the influenza surveillance network in 1952 to monitor the continuous antigenic changes of the virus and to guide vaccine composition. Following the 2009 influenza pandemic, the scope of the surveillance was extended to better assess the severity and burden of influenza viruses. In Belgium, influenza virus surveillance is organised by the national public health institute, Sciensano, which also hosts the National Influenza Centre (NIC). Two sentinel networks are in place: (i) surveillance of influenza-like illness (ILI) in the community, based on general practitioners (GPs) providing information on mild cases (in place since the mid-1970s) and (ii) surveillance of severe acute respiratory infection (SARI) based on six sentinel hospitals providing information on hospitalised patients (set up after the 2009 influenza pandemic and operating every season since 2011). Starting with the 2015/16 influenza season, the NIC introduced systematic testing of NIRV by qPCR in addition to testing and characterising influenza viruses. We report here the results of systematic NIRV testing of ILI and SARI surveillance samples for four seasons (2015/16 to 2018/19) and estimate the burden and severity of NIRV compared with influenza.

\section{Methods}

\section{Settings, participants and variables}

Sentinel-based ILI surveillance was performed through a network of GPs widely distributed throughout Belgium, representing ca $1.5 \%$ of all Belgian GPS and covering more than $1.1 \%$ of the population. Patients were enrolled during each influenza season surveillance period (from week 40 to week 20). Recommendations were that nasopharyngeal swabs were obtained weekly from the first two ILI patients belonging to two different households. SARI patients were recruited through the sentinel network of six hospitals in Belgium, two in each administrative region of the country: Flanders, Wallonia and Brussels-Capital. It was recommended that paediatric and adult units systematically collect detailed clinico-epidemiological data and a respiratory specimen from all patients meeting the SARI case definition during the epidemic period of seasonal influenza (starting and ending weeks between January and April, depending on influenza activity).

Based on WHO guidelines [10], ILI and SARI cases were defined as acute respiratory illness with onset within the last 10 days, with measured or reported fever of $\geq 38^{\circ} \mathrm{C}$ and with cough and/or dyspnoea. In addition, overnight hospitalisation was a required criterion for SARI cases. Patients who did not give informed consent (either directly or through a parent or legal guardian) were not enrolled in the study nor sampled.

Standardised questionnaires were used to collect data on age, sex, clinical signs included in the case definition, status of vaccination against influenza viruses, administration of a neuraminidase inhibitor antiviral and/or antibiotic treatment, and co-morbidities known as risk factors of severity. Follow-up data during hospitalisation were also reported for the SARI cases to evaluate disease severity and included the detection of pneumonia based on chest radiography, and/or the development of acute respiratory distress syndrome, and/or the requirement for respiratory assistance and/ or for extracorporeal membrane oxygenation, and/or the admission to an intensive care unit, and/or death (all-cause death). 


\section{FIGURE 2}

Proportions of the different respiratory viruses detected by multiplex RT-qPCR per season and overall, Belgium, 2015-2019 $(\mathrm{n}=6,565)$

A. Influenza-like illness

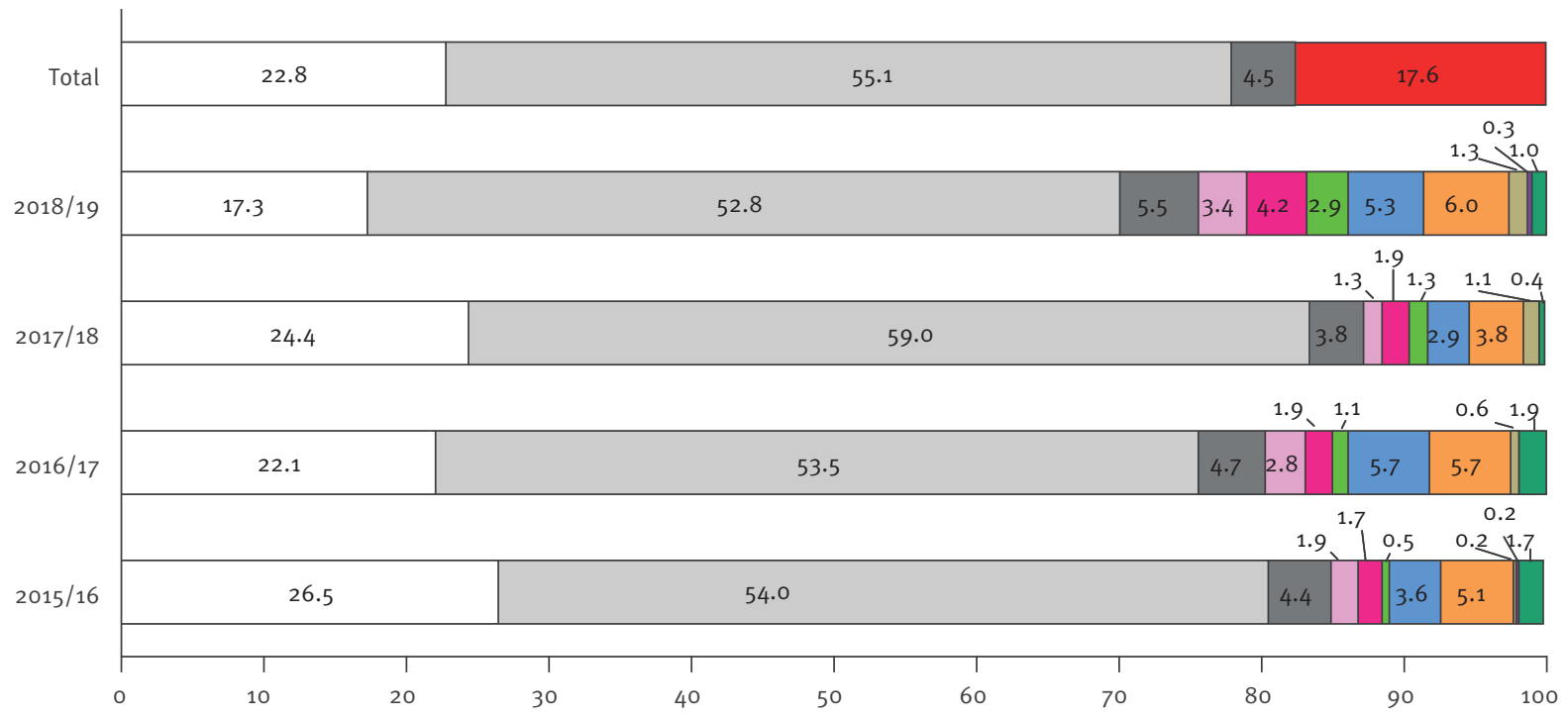

B. Severe acute respiratory infection

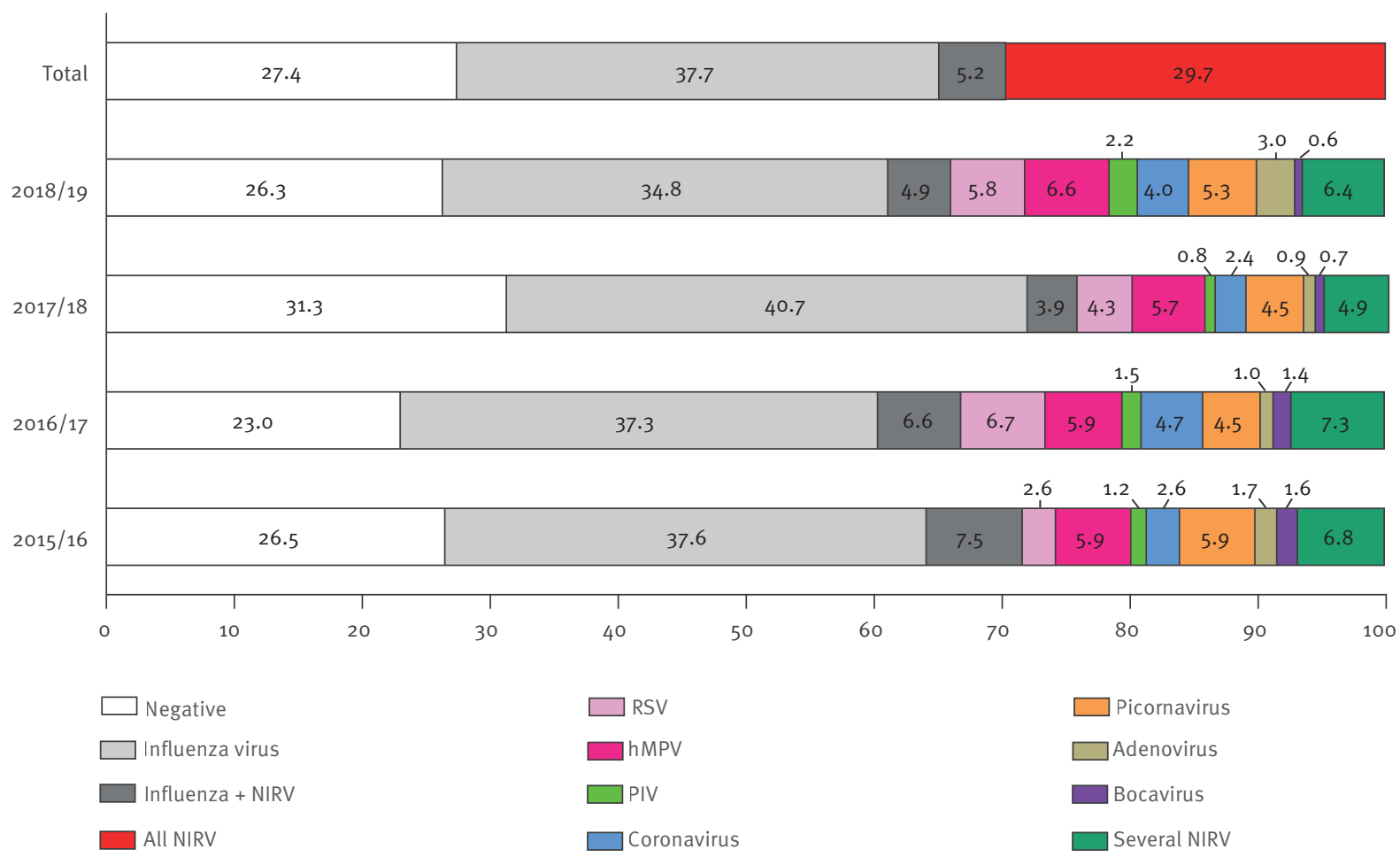

Coronavirus: coronavirus CoV-OC43, CoV-NL63 or CoV-229E; hMPV: human metapneumovirus; influenza virus: influenza virus type A or B; influenza + NIRV: co-detection of influenza virus with at least one non-influenza respiratory virus; negative: negative for all tested viruses; NIRV: positive for non-influenza respiratory virus(es); picornavirus: picornavirus of the Rhinovirus and Enterovirus genera or parechovirus; PIV: parainfluenzavirus type 1, 2, 3 or 4; RSV: respiratory syncytial virus type A or B; several NIRV: co-detection of several non-influenza respiratory viruses. 


\section{Laboratory testing}

Respiratory samples were analysed at the Belgian National Influenza Centre. Viral nucleic acids were extracted using NucliSENS EasyMag (BioMerieux Benelux, Brussels, Belgium). The following respiratory virus targets were detected using several in-house multiplex qPCRs: influenza virus types A and B (and subsequent subtype/lineage), respiratory syncytial virus types $A$ and $B$, human metapneumoviruses, parainfluenzavirus types $1,2,3$ and 4, coronaviruses CoV-OC43, CoV-NL63 and CoV-229E, adenoviruses, picornaviruses (Rhinovirus and Enterovirus genera), specific enterovirus D68, parechovirus and bocavirus as previously described [11].

\section{Incidence rates of virus-associated severe acute respiratory infection}

To evaluate some aspects of the burden of disease of virus-associated SARI, the catchment population estimates from 2017 (mid-year of the study period) were used to calculate monthly incidence rate per 100,000 population during the winter season, based on WHO recommendations [10]. Catchment populations of the SARI hospitals by age group were estimated based on hospital admission data (i.e. the proportion of the different municipalities) and the total population of these municipalities; this rough estimation has the advantage that it is easily extracted without extra workload for the hospitals. The number of months covered by the study period (i.e. 14.9 months), used for the denominator, was calculated by adding up the number of weeks with active SARI surveillance for each season (i.e. 64 weeks), multiplied by 7 days and divided by the average number of days in a month during the winter period (December to April, i.e. 30 days). Age groupspecific catchment populations of the six hospitals were summed up to calculate the monthly incidence rate by age group $(<5,5-14,15-64$ and $\geq 65$ years) for virus-associated SARI. The $95 \%$ confidence intervals (CI) were calculated using the Rothman-Greenland method [12].

\section{Ethical statement}

The SARI surveillance protocol was approved by a central Ethical Committee (reference AK/12-02-11/4111; in 2011: Centre Hospitalier Universitaire St-Pierre, Brussels, Belgium; from 2014 onwards: Universitair Ziekenhuis Brussel, Brussels, Belgium) and the local ethical committees of each participating hospital. Informed consent was obtained from all participants or parents/guardians.

\section{Results}

\section{Study population}

Over the four seasons under study 1,791 ILI (Figure $1 \mathrm{~A}$ ) and 4,774 SARI (Figure 1B) patients responding to the case definition were retained in the analysis. Among the ILI patients with known age, $78.7 \%$ were adults (15-64 years-old, 1,356/1,724), 14.2\% were children (\&15 years-old, 244/1,724) and $7.2 \%$ were older adults (65 years and older, 124/1,724). Half were female $(833 / 1,692)$, including 11 who were pregnant. Among the SARI patients, there were slightly more male than female patients $(52.2 \% ; 2,428 / 4,650)$, and 28 women were pregnant. Children and older adults represented, respectively, 35.5\% (1,689/4,762) and $44.1 \%(2,099 / 4,762)$ of the SARI cases with known age. Among children, $44.6 \%$ and $42.1 \%$ were younger than 1 year $(753 / 1,689)$ and between 1 and 4 years-old $(711 / 1,689)$, respectively. The six sentinel hospitals contributed heterogeneously to the distribution of SARI patients in terms of age, with one hospital reporting more paediatric cases (Supplementary Table S1).

\section{Overall respiratory virus detection rates}

Among the 1,791 ILI patients, 408 (22.8\%) were negative for all respiratory viruses tested, 1,068 (59.6\%) were positive for influenza virus (of which 81 were codetections with NIRV), and 315 (17.6\%) were negative for influenza virus but positive for NIRV (of which 22 were co-detections of several NIRV) (Figure 2A). Among the 4,774 SARI patients, 1,309 (27.4\%) were negative for all respiratory viruses tested, 2,049 (42.9\%) were positive for influenza virus (of which 250 were co-detections with NIRV) and 1,415 (29.6\%) were negative for influenza virus but positive for at least one NIRV (with 291 being co-detections with several NIRV) (Figure 2B).

All seasons taken together, the most common single NIRV detections among ILI patients were picornaviruses (5.1\%; 91/1,791) and coronaviruses (4.3\%; 77/1,791), followed by human metapneumoviruses (hMPV) (2.3\%; $42 / 1,791$ ) and respiratory syncytial viruses (RSV) $(2.3 \% ; 41 / 1,791)$. Single detections of parainfluenza virus, adenovirus and bocavirus accounted for $1.4 \%$, $0.8 \%$ and $0.1 \%$, respectively (Supplementary Table S2). Among SARI patients, the main single NIRV detections were hMPV $(6.1 \% ; 291 / 4,774)$, RSV (5.0\%; 238/4,774), picornaviruses $(5.0 \% ; 237 / 4,774)$ and coronaviruses $(3.4 \% ; 162 / 4,774)$. Single detections of parainfluenza virus, adenovirus and bocavirus accounted for $1.4 \%$, $1.7 \%$ and $0.9 \%$, respectively (Supplementary Table S2). Co-detections were more common among SARI patients than ILI patients. They accounted for $11.3 \%$ of the overall number of tested SARI samples $(541 / 4,774)$, with influenza virus-positive $(n=250)$ and influenzanegative $(n=291)$ co-detections representing 5.2 and $6.1 \%$, respectively.

The proportion of NIRV detection (excluding co-infections with influenza viruses) varied between $12.8 \%$ in $2017 / 18$ and $24.3 \%$ in $2018 / 19$ in the ILI samples (Figure 2A). In SARI patients, the proportion was higher and varied between $24.2 \%$ in 2017/18 and $33.9 \%$ in 2018/19 (Figure 2B). Within a season, the proportions of picornaviruses and coronaviruses were similar among ILI and SARI patients. On the contrary, hMPV and RSV detection rates were systematically higher in SARI than in ILI samples. Except for season $2015 / 16$, these two viruses represented the largest groups in SARI patients, whereas picornaviruses and 


\section{FIGURE 3}

Distribution of the positive samples for different respiratory viruses by season and sampling week for patients with severe acute respiratory infection, Belgium, 2015-2019 $(\mathrm{n}=4,774)$

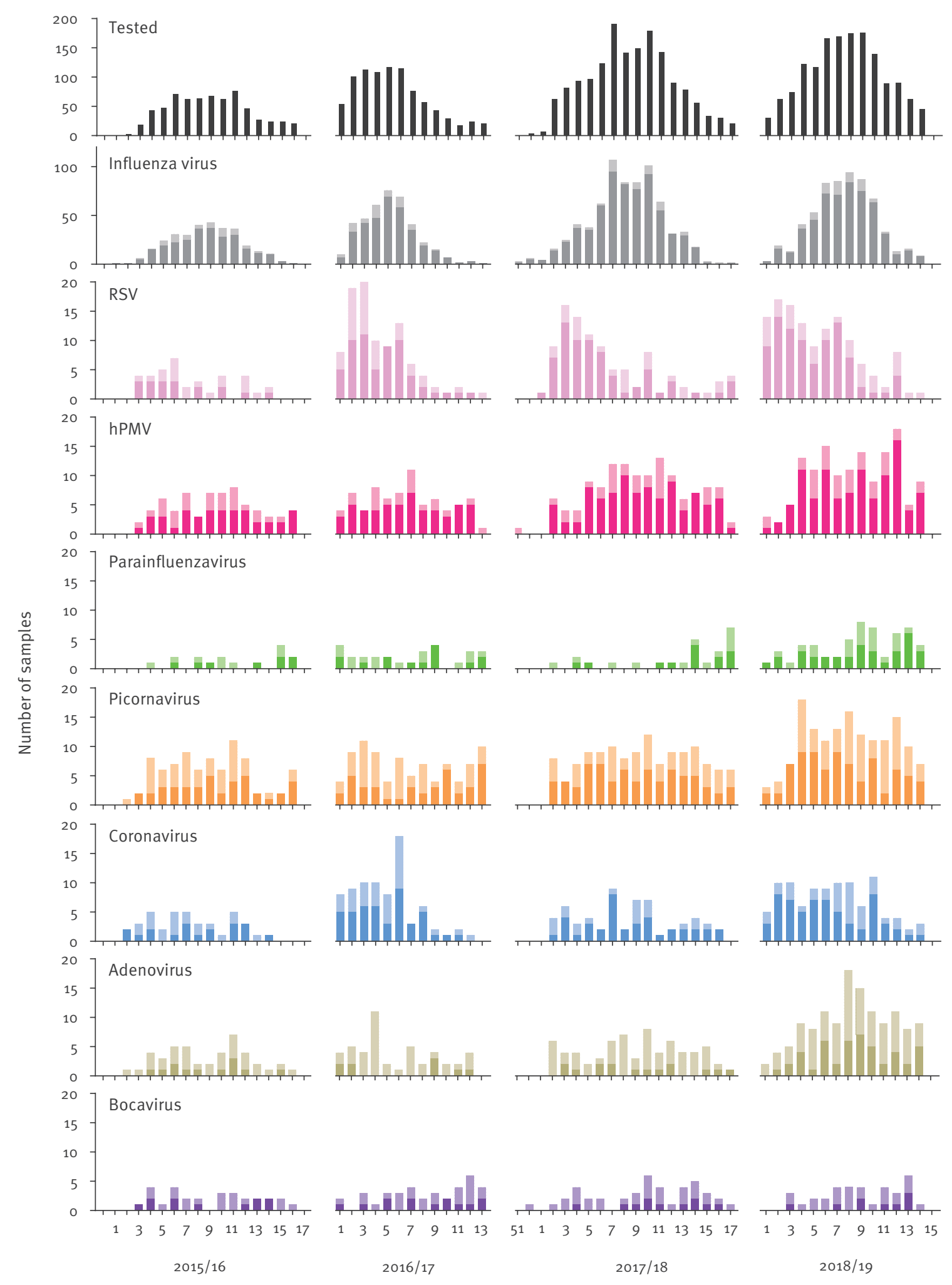

hPMV: human metapneumovirus; RSV: respiratory syncytial virus.

Colour codes as in Figure 2. Solid colour: single detection; shaded colour: in co-detection with at least one other virus. The x-axes include weeks of active SARI surveillance: $2015 / 16$, week 1 to week 17 of 2016; 2016/17, week 1 to week 13 of 2017 ; $2017 / 18$, week 51 of 2017 to week 17 of 2018; 2018/19, week 1 to week 15 of 2019 
Respiratory virus single and co-detection in influenza-like illness and severe acute respiratory infection surveillance patients by age group, Belgium, 2015-2019 $(n=6,565)$

\begin{tabular}{|c|c|c|c|c|c|c|c|c|c|}
\hline & \multicolumn{2}{|c|}{ Children } & \multicolumn{2}{|c|}{ Adults } & \multicolumn{2}{|c|}{ Older adults } & \multirow{2}{*}{$\begin{array}{c}\text { Missing age } \\
n\end{array}$} & \multicolumn{2}{|c|}{ Total } \\
\hline & $\mathrm{n}$ & $\%$ & $\mathrm{n}$ & $\%$ & $\mathrm{n}$ & $\%$ & & $\mathrm{n}$ & $\%$ \\
\hline \multicolumn{10}{|l|}{ ILI } \\
\hline Overall & \multicolumn{2}{|c|}{244} & \multicolumn{2}{|c|}{1,364} & \multicolumn{2}{|c|}{124} & 59 & \multicolumn{2}{|c|}{1,791} \\
\hline Negative & 33 & 13.5 & 327 & 24.0 & 29 & 23.4 & 19 & 408 & 22.8 \\
\hline Single influenza & 158 & 64.8 & 737 & 54.0 & 64 & 51.6 & 28 & 987 & 55.1 \\
\hline Influenza + NIRV & 15 & 6.1 & 58 & $4 \cdot 3$ & 6 & 4.8 & 2 & 81 & 4.5 \\
\hline Total influenza & 173 & 70.9 & 795 & 58.3 & 70 & 56.5 & 30 & 1,068 & 59.6 \\
\hline RSV & 4 & 1.6 & 33 & 2.4 & 3 & 2.4 & 1 & 41 & 2.3 \\
\hline hMPV & 8 & $3 \cdot 3$ & 30 & 2.2 & 3 & 2.4 & 1 & 42 & 2.3 \\
\hline Parainfluenza & 3 & 1.2 & 16 & 1.2 & 4 & 3.2 & 2 & 25 & 1.4 \\
\hline Coronavirus & 7 & 2.9 & 61 & $4 \cdot 5$ & 9 & $7 \cdot 3$ & 0 & 77 & $4 \cdot 3$ \\
\hline Picornavirus & 7 & 2.9 & 79 & 5.8 & 3 & 2.4 & 2 & 91 & 5.1 \\
\hline Adenovirus & 3 & 1.2 & 9 & 0.7 & 1 & 0.8 & 2 & 15 & 0.8 \\
\hline Bocavirus & 0 & 0.0 & 2 & 0.1 & 0 & 0.0 & 0 & 2 & 0.1 \\
\hline Total single NIRV & 32 & 13.1 & 230 & 16.9 & 23 & 18.5 & 8 & 293 & 16.4 \\
\hline NIRV coinfection & 6 & 2.5 & 12 & 0.9 & 2 & 1.6 & 2 & 22 & 1.2 \\
\hline Total NIRV & 38 & 15.6 & 242 & $17 \cdot 7$ & 25 & 20.2 & 10 & 315 & 17.6 \\
\hline \multicolumn{10}{|l|}{ SARI } \\
\hline Overall & \multicolumn{2}{|c|}{1,677} & \multicolumn{2}{|c|}{977} & \multicolumn{2}{|c|}{2,105} & 15 & \multicolumn{2}{|c|}{4,774} \\
\hline Negative & 302 & 18.0 & 373 & 38.2 & 631 & 30.0 & 4 & 1,310 & 27.4 \\
\hline Single influenza & 407 & $24 \cdot 3$ & 390 & 39.9 & 996 & $47 \cdot 3$ & 6 & 1,799 & 37.7 \\
\hline Influenza + NIRV & 140 & 8.3 & 32 & $3 \cdot 3$ & 78 & $3 \cdot 7$ & 0 & 250 & 5.2 \\
\hline Total influenza & 547 & 32.6 & 422 & 43.2 & 1,074 & 51.0 & 6 & 2,049 & 42.9 \\
\hline RSV & 105 & 6.3 & 33 & 3.4 & 100 & 4.8 & 0 & 238 & 5.0 \\
\hline hMPV & 130 & 7.8 & 48 & 4.9 & 112 & $5 \cdot 3$ & 1 & 291 & 6.1 \\
\hline Parainfluenza & 40 & 2.4 & 8 & 0.8 & 20 & 1.0 & 1 & 69 & 1.4 \\
\hline Coronavirus & 40 & 2.4 & 39 & 4.0 & 82 & 3.9 & 1 & 162 & 3.4 \\
\hline Picornavirus & 151 & 9.0 & 33 & 3.4 & 52 & 2.5 & 1 & 237 & 5.0 \\
\hline Adenovirus & 63 & 3.8 & 10 & 1.0 & 10 & 0.5 & 0 & 83 & 1.7 \\
\hline Bocavirus & 44 & 2.6 & 0 & 0.0 & 0 & 0.0 & 0 & 44 & 0.9 \\
\hline Total single NIRV & 573 & 34.2 & 171 & 17.5 & 376 & 17.9 & 4 & 1,124 & 23.5 \\
\hline NIRV coinfection & 255 & 15.2 & 11 & 1.1 & 24 & 1.1 & 1 & 291 & 6.1 \\
\hline Total NIRV & 828 & 49.4 & 182 & 18.6 & 400 & 19.0 & 5 & 1,415 & 29.6 \\
\hline
\end{tabular}

Coronavirus: coronavirus CoV-OC43, CoV-NL63 or CoV-229E; hMPV: human metapneumovirus; ILI: influenza-like illness; influenza + NIRV: co-detection of influenza virus with at least one non-influenza respiratory virus; NIRV: non-influenza respiratory virus; NIRV coinfection: co-detection of several non-influenza respiratory viruses; parainfluenza: parainfluenzavirus type 1, 2, 3 or 4; picornavirus: picornavirus of the Rhinovirus and Enterovirus genera or parechovirus; RSV: respiratory syncytial virus type A or B; SARI: severe acute respiratory infection; Single influenza: influenza virus type A or B only.

Age groups: children, < 15 years; adults, 15-64 years old; older adults, $\geq 65$ years; missing, unknown age.

Percentage per column, calculated based on the overall number. Not calculated for 'missing' groups.

coronaviruses were the most frequent ones in the ILI patients. Season 2018/19 was marked by higher detection rates for parainfluenza viruses (both in ILI and SARI) and adenoviruses (in SARI) compared with previous seasons (Figure 2, Supplementary Table S2).

Both ILI and SARI surveillances were able to capture the seasonal circulation of influenza virus, but such bell-shape epidemic curves were not detected for other NIRV, with perhaps two exceptions: coronaviruses in the ILI surveillance during season 2016/17 despite the limited number of positive samples (Supplementary Figure S1) and RSV in the SARI surveillance, with the detection of the end of the RSV epidemic, which usually occurs before the influenza epidemic in western Europe [13] including in Belgium [14] (Figure 3). 


\section{FIGURE 4}

Incidence rates of virus-associated severe acute respiratory infections per 100,000 person-months by age group, Belgium, 2015-2019 $(\mathrm{n}=4,774)$

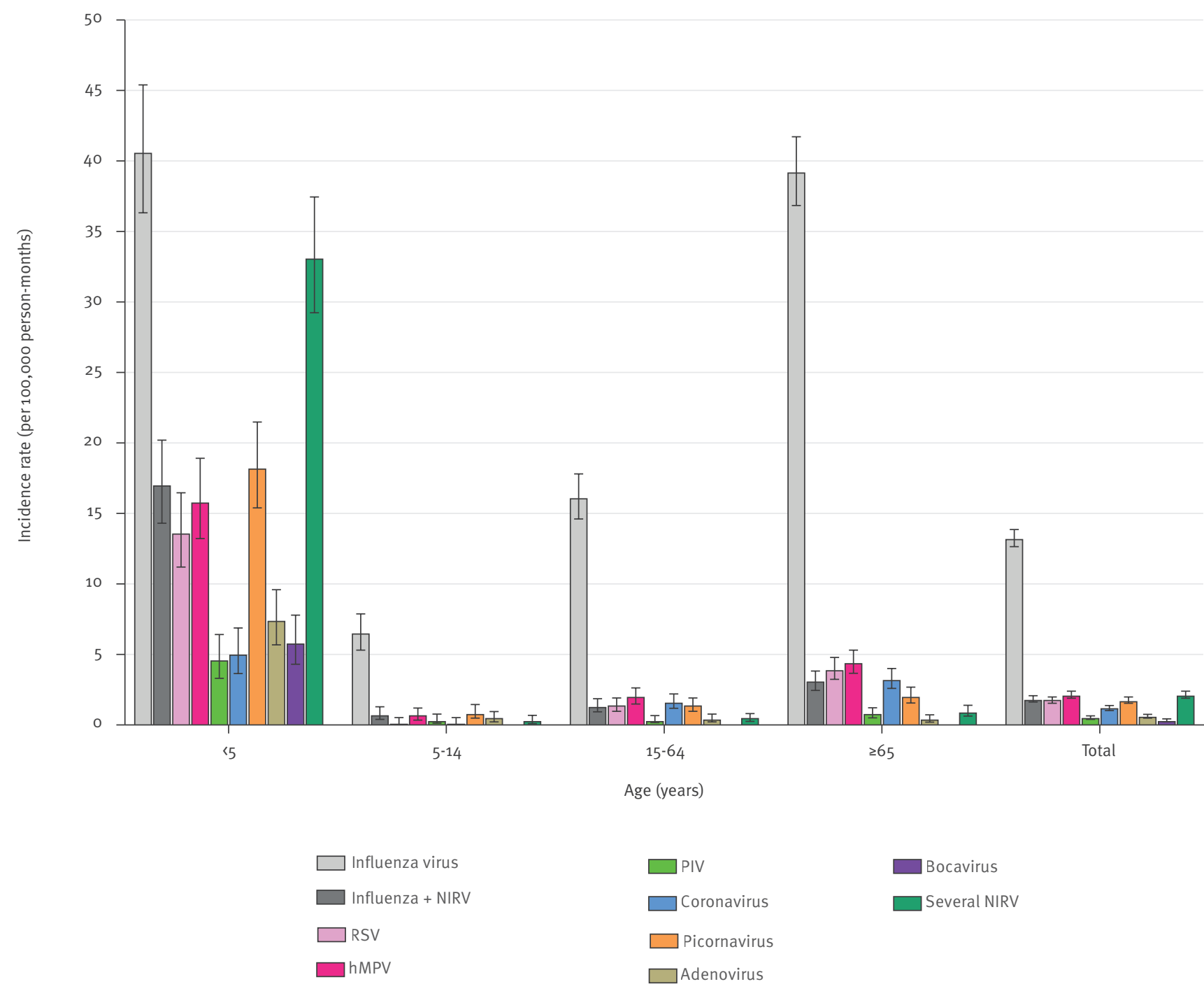

Coronavirus: coronavirus CoV-OC43, CoV-NL63 or CoV-229E; hMPV: human metapneumovirus; influenza virus: influenza virus type A or $\mathrm{B}$; influenza + NIRV: co-detection of influenza virus with at least one non-influenza respiratory virus; picornavirus: picornavirus of the Rhinovirus and Enterovirus genera or parechovirus; PIV: parainfluenzavirus type 1, 2, 3 or 4; RSV: respiratory syncytial virus type A or B; several NIRV: co-detection of several non-influenza respiratory viruses.

\section{Age-specific detection rates of non-influenza} respiratory viruses and NIRV-associated SARI incidence rates

In children (younger than 15 years), the proportion of samples positive for NIRV (single or co-detection) was higher in SARI than ILI patients (Table). The proportion positive for NIRV only (excluding co-detection with influenza viruses) was $49.4 \%(828 / 1,677)$ in children with SARI and only $15.6 \%(38 / 244)$ in children with ILI (Fisher's exact test, $p<0.001$ ). In contrast, the proportions of positive NIRV samples among adults (15-64 years-old) and older adults ( $\geq 65$ years-old) taken together were similar for ILI and SARI surveillance
(17.9\%; 267/1,488 and $18.9 \% ; 582 / 3,082$, respectively; Fisher's exact test, $p=0.465$ ).

Half $(82 / 162)$ of the SARI samples positive for coronavirus only were from older adults (Table). On the contrary, all SARI samples positive for bocavirus only $(n=44)$ and $75.9 \%(63 / 83)$ of those positive for adenovirus only were from children. The SARI samples positive for RSV only and hMPV only were from both children (44.1\%; 105/238 and 44.7\%; 130/291, respectively), mainly younger than 1 year, and from older adults ( $42.0 \% ; 100 / 238$ and $38.5 \% ; 112 / 291$, respectively). Picornavirus-only and parainfluenzavirus-only SARI samples were mainly from children under the age of 5 years $(58.2 \% ; 138 / 237$ and $50.7 \% ; 35 / 69$, 
respectively, Supplementary Table S3), but also from older adults (21.9\%; 52/237 and $29.0 \% ; 20 / 69$, respectively). Finally, SARI samples with co-detection of NIRV or of NIRV with influenza viruses were largely from children younger than 5 years $(86.3 \% ; 251 / 291$ and $51.6 \%$; 129/250, respectively, Supplementary Table S3).

The burden of NIRV captured by influenza surveillance was partially evaluated by calculating the incidence rates of SARI associated with each virus within specific age groups and for all the samples (Figure 4 and Supplementary Table S4). NIRV incidence rates were noticeably higher in the youngest age group ( 15 years) than in the other age groups or the overall population, with RSV, hMPV and picornaviruses contributing the most as single infection (13.6, 15.8 and 18.2 per 100,000 person-months, respectively), followed by adenoviruses, bocavirus, coronaviruses and parainfluenzaviruses $(7.4,5.8,5.0$ and 4.6 per 100,000 personmonths, respectively). In this age group, the incidence rate of SARI associated with all kinds of influenza-negative NIRV infections was almost double that associated with influenza (103.5 VS 57.6 per 100,000 personmonths). Incidence rates of SARI associated with NIRV were low in the other age groups, in contrast to those associated with influenza (Figure 4). Despite that, the incidence rates of SARI associated with RSV, hMPV and coronaviruses in older adults were more than twice that in the overall population (3.9, 4.4 and 3.2 vs 1.8 , 2.1 and 1.2 per 100,000 person-months, respectively).

\section{Discussion}

Here, we report on the use of several in-house multiplex qPCR assays on respiratory specimens collected through two sentinel networks of influenza surveillance (ILI and SARI) to detect a broad range of respiratory viruses of public health relevance during the winter season. Systematic testing of NIRV on samples was introduced in Belgium from the winter season 2015/16 onwards, with the use of in-house multiplex qPCRs offering the flexibility to change the panel of viruses/virus groups depending on the situation. We found that NIRV testing may explain up to one third of infections and becomes particularly useful for diagnostic purposes of paediatric SARI patients. By estimating incidence rates, we showed that NIRV even represent a higher burden than influenza viruses in the SARI patients under the age of 5 years.

A limitation of our study is that these sentinel surveillance networks collect data only during the winter season in Belgium. This is particularly true for the SARI network which operates only during the period of influenza virus activity. In addition, since the objective of the SARI surveillance is primarily to evaluate the severity of influenza viruses, the definition used might not be optimally adapted for NIRV. The criteria 'fever' and 'cough' were chosen to specifically target influenza viruses [15], but they are also characteristic of most NIRV, including RSV and hMPV [16], the two main NIRV identified in our study. Despite the differences in the case definition and the period of surveillance, our results remain comparable to those obtained in other countries regarding the NIRV identified and their proportions $[17,18]$, but the detection rates observed here for the different NIRV might not represent their whole circulation patterns. For example, it was shown that the intensive circulation period for RSV usually precedes that of influenza viruses in western Europe [13]. Although RSV, hMPV, coronaviruses and other NIRV do not strictly follow the same pattern as influenza viruses [19], many tend to also circulate during the winter season in temperate regions. Interactions between respiratory viruses have been reported $[20,21]$, including a negative association between rhinovirus and RSV infection in children [22]. There have also been reports of an increased risk of NIRV infections in children who received inactivated influenza vaccine in a small-scale study [23], although this was not observed in a larger study encompassing data from seven seasons [24]. Influenza sentinel surveillance thus appears as a relevant system for the study of NIRV $[25,26]$ and we consider that extending the period of SARI surveillance in Belgium would be beneficial to better estimate their burden but also, on a long-term perspective, the potential impact of the new severe acute respiratory syndrome coronavirus 2 after the current pandemic phase, if it establishes itself as a seasonal virus.

Another limitation is that paediatric and geriatric samples are underrepresented in ILI patients, probably due to sampling bias (GPs in Belgium being less likely to have children or older people as patients or to sample them).

The systematic testing for NIRV allowed the detection of a pathogen in only one eighth of the ILI patients, suggesting that the additional effort of testing NIRV in this study population may be of limited interest and not cost-effective. In contrast, samples were NIRV-positive in almost a third of the SARI patients, in whom the morbidity and mortality associated with respiratory infection is the highest, and in almost half of the paediatric SARI patients. The burden associated with NIRV in SARI patients was age-dependent and particularly high for young children ( 55 years). While co-infections with several NIRV were rarely detected among ILI patients, they were almost as common as influenza virus infection among paediatric SARI patients younger than 5 years. The non-negligible burden associated with RSV should also be taken into consideration in the coming years, when RSV vaccines become available and vaccination is implemented [27]. This clearly shows the benefit that testing SARI patients for NIRV infections can have on the surveillance trend analysis but also, to a lesser extent, on clinical management.

Despite an important decrease in the number of specimens that were reported as negative in SARI patients following the introduction of NIRV testing, more than one fourth of SARI patients remained negative for all tested viruses. Although viruses are considered as 
the main cause of acute respiratory tract infections [28-30] and our methodology covers the main respiratory viruses that are involved [31], it is possible that some samples were positive for viruses that were not targeted by our multiplex qPCRs (e.g. coronavirus HKU1 , influenza $C$ virus, cytomegalovirus, herpes simplex virus), or for bacterial or fungal respiratory pathogens, known to substantially contribute to complications in lower respiratory tract infections [32-34]. In a prospective study in 11 European countries that looked at the aetiology of lower respiratory tract infections among adults, a bacterial pathogen was detected in one fifth of the participants [35]. The difference in the proportion of 'negative' samples that we observed between age groups (one third among adults and one fifth among children) suggests that the pathogens causing SARI differ between children (more broadly covered by our multiplex PCRs) and adults, which could be due to the clinical signs of the SARI case definition being less specific for adults, especially older adults $[36,37]$.

\section{Conclusion}

Our study demonstrates the added value of testing NIRV with a flexible method and confirms that the burden of disease associated with NIRV in SARI patients should not be underestimated, especially in children under the age of 5 years for whom it is even higher than that of influenza viruses. Although more expensive than other surveillance systems, SARI sentinel surveillance offers the advantage that it relies on a precise case definition and collects data on complications and co-morbidities. Throughout the years, as data obtained in a standardised manner accumulate, such system could provide better estimates of the burden of NIRV.

\section{Acknowledgements}

The authors would like to thank Jeannine Weyckmans, Ilham Fdillate, Assia Hamouda and Reinout Van Eycken from the NIC for their excellent technical assistance throughout the surveillance seasons; Evelyn Petit from AZ Sint-Jan, Françoise Antoine, Tiphaine Mouquet and Christelle Nguepi from CHU St-Pierre, Lore-Lien Roels and Prof. Elke De Wachter from UZ Brussels, Marlies Blommen, Annemie Forier and Dr. Luc Waumans from Jessa Ziekenhuis, Catherine Sion and Mohamed El Kaissi from Grand Hôpital de Charleroi for their dedication in the follow-up of the SARI cases; and all the doctors and nurses of the six hospitals who took part in the recruitment of the SARI cases, without whom the SARI surveillance would not be possible. N.D. is a Post-doctorate Clinical Master Specialist of the FRS-FNRS (Fond National de la Recherche Scientifique).

Funding: This work was supported by the Belgian Federal Public Service 'Health, Food Chain Safety, and Environment', the Belgian National Insurance Health Care (INAMI/RIZIV), and the Regional Health Authorities of Flanders (Agentschap Zorg en Gezondheid, AZG), of Brussels (Commission communautaire commune de Bruxelles-Capitale, COCOM), and of Wallonia (Agence pour une Vie de Qualité, AVIQ).

\section{Conflict of interest}

None declared.
Authors' contributions

Project administration: C.B., N.B., I.T. Data curation: L.S., C.B., N.B., I.T. Formal analysis: L.S., C.B. Investigation: M.R., M.G., N.D., P.L., S.D., B.L., X.H., K.M., D.J., M.B., B.D., C.B., I.T. Resources: M.R., M.G., N.D., P.L., S.D., B.L., X.H., K.M., D.J., M.B., B.D. Visualisation: L.S., C.B. Writing - original draft preparation: L.S., C.B. Writing - review and editing: all authors. Funding acquisition: S.Q., S.V.G.

\section{References}

1. Forum of International Respiratory Societies. The global impact of respiratory disease - Second edition. Sheffield: European Respiratory Society; 2017. Available from: https://www.who. int/gard/publications/The_Global_Impact_of_Respiratory_ Disease.pdf

2. Malosh RE, Martin ET, Ortiz JR, Monto AS. The risk of lower respiratory tract infection following influenza virus infection: A systematic and narrative review. Vaccine. 2018;36(1):141-7. https://doi.org/10.1016/j.vaccine.2017.11.018 PMID: 29157959

3. Fendrick AM, Monto AS, Nightengale B, Sarnes M. The economic burden of non-influenza-related viral respiratory tract infection in the United States. Arch Intern Med. 2003;163(4):487-94. https://doi.org/10.1001/ archinte.163.4.487 PMID: 12588210

4. Gilca R, Amini R, Douville-Fradet M, Charest H, Dubuque J, Boulianne $\mathrm{N}$, et al. Other respiratory viruses are important contributors to adult respiratory hospitalizations and mortality even during peak weeks of the influenza season. Open Forum Infect Dis. 2014;1(2):ofuo86. https://doi.org/10.1093/ofid/ ofuo86 PMID: 25734152

5. Furuta T, Hasegawa S, Mizutani M, Iwai T, Ohbuchi N, Kawano $S$, et al. Burden of human metapneumovirus and respiratory syncytial virus infections in asthmatic children. Pediatr Infect Dis J. 2018;37(11):1107-11. https://doi.org/10.1097/ INF.0000000000002038 PMID: 29742646

6. Taylor S, Lopez P, Weckx L, Borja-Tabora C, Ulloa-Gutierrez R, Lazcano-Ponce E, et al. Respiratory viruses and influenza-like illness: Epidemiology and outcomes in children aged 6 months to 10 years in a multi-country population sample. J Infect. 2017;74(1):29-41. https://doi.org/10.1016/j.jinf.2016.09.003 PMID: 27667752

7. Uddin SMI, Englund JA, Kuypers JY, Chu HY, Steinhoff MC, Khatry SK, et al. Burden and risk factors for coronavirus infections in infants in rural Nepal. Clin Infect Dis. 2018;67(10):1507-14. https://doi.org/10.1093/cid/ciy317 PMID: 29668900

8. Bénézit F, Loubet P, Galtier F, Pronier C, Lenzi N, Lesieur Z, et al. , FLUVAC Study Group. Non-influenza respiratory viruses in adult patients admitted with influenza-like illness: a 3-year prospective multicenter study. Infection. 2020;48(4):489-95. https://doi.org/10.1007/s15010-019-01388-1 PMID: 32056143

9. Nair H, Nokes DJ, Gessner BD, Dherani M, Madhi SA, Singleton RJ, et al. Global burden of acute lower respiratory infections due to respiratory syncytial virus in young children: a systematic review and meta-analysis. Lancet. 2010;375(9725):1545-55. https://doi.org/10.1016/S01406736(10)60206-1 PMID: 20399493

10. World Health Organization (WHO). A manual for estimating disease burden associated with seasonal influenza. Geneva: WHO; 2015. Available from: https://apps.who.int/iris/ bitstream/handle/10665/178801/9789241549301_eng.pdf;jses sionid=AECA0159A38911F4578CACA12131DD04? sequence $=1$

11. Fischer N, Dauby N, Bossuyt N, Reynders M, Gérard M, Lacor $P$, et al. Monitoring of human coronaviruses in Belgian primary care and hospitals, 2015-20: a surveillance study. Lancet Microbe. 2021;2(3):e105-14. https://doi.org/10.1016/S26665247(20)30221-4 PMID: 33937883

12. Rothman KJ, Greenland S. Modern epidemiology. 2nd edition. Philadelphia: Lippincott-Raven, 1998.

13. Broberg EK, Waris M, Johansen K, Snacken R, Penttinen P, European Influenza Surveillance Network. Seasonality and geographical spread of respiratory syncytial virus epidemics in 15 European countries, 2010 to 2016 . Euro Surveill. 2018;23(5):17-00284. https://doi.org/10.2807/1560-7917. ES.2018.23.5.17-00284 PMID: 29409569

14. Subissi L, Bossuyt N, Reynders M, Gérard M, Dauby N, Bourgeois $M$, et al. Capturing respiratory syncytial virus season in Belgium using the influenza severe acute respiratory infection surveillance network, season 2018/19. Euro Surveill. 2020;25(39):1900627. https://doi.org/10.2807/1560-7917. ES.2020.25.39.1900627 PMID: 33006303 
15. Monto AS, Gravenstein S, Elliott M, Colopy M, Schweinle J. Clinical signs and symptoms predicting influenza infection. Arch Intern Med. 2000;160(21):3243-7. https://doi. org/10.1001/archinte.160.21.3243 PMID: 11088084

16. Ma X, Conrad T, Alchikh M, Reiche J, Schweiger B, Rath B. Can we distinguish respiratory viral infections based on clinical features? A prospective pediatric cohort compared to systematic literature review. Rev Med Virol. 2018;28(5):e1997. https://doi.org/10.1002/rmv.1997 PMID: 30043515

17. Kurskaya O, Ryabichenko T, Leonova N, Shi W, Bi H, Sharshov $\mathrm{K}$, et al. Viral etiology of acute respiratory infections in hospitalized children in Novosibirsk City, Russia (2013 - 2017). PLoS One. 2018;13(9):e0200117. https://doi.org/10.1371/ journal.pone.0200117 PMID: 30226876

18. Chakhunashvili G, Wagner AL, Power LE, Janusz CB, Machablishvili A, Karseladze I, et al. Severe Acute Respiratory Infection (SARI) sentinel surveillance in the country of Georgia, 2015-2017. PLoS One. 2018;13(7):e0201497. https://doi. org/10.1371/journal.pone.0201497 PMID: 30059540

19. Li Y, Reeves RM, Wang X, Bassat Q, Brooks WA, Cohen C, et al. , RSV Global Epidemiology Network, RESCEU investigators. Global patterns in monthly activity of influenza virus, respiratory syncytial virus, parainfluenza virus, and metapneumovirus: a systematic analysis. Lancet Glob Health. 2019;7(8):e1031-45. https://doi.org/10.1016/S2214109X(19)30264-5 PMID: 31303294

20. van Asten L, Bijkerk P, Fanoy E, van Ginkel A, Suijkerbuijk $A$, van der Hoek W, et al. Early occurrence of influenza $A$ epidemics coincided with changes in occurrence of other respiratory virus infections. Influenza Other Respir Viruses. 2016;10(1):14-26. https://doi.org/10.1111/irv.12348 PMID: 26369646

21. Price OH, Sullivan SG, Sutterby C, Druce J, Carville KS. Using routine testing data to understand circulation patterns of influenza $A$, respiratory syncytial virus and other respiratory viruses in Victoria, Australia. Epidemiol Infect. 2019;147:e221. https://doi.org/10.1017/So950268819001055 PMID: 31364539

22. Achten NB, Wu P, Bont L, Blanken MO, Gebretsadik T, Chappell JD, et al. Interference between respiratory syncytial virus and human rhinovirus infection in Infancy. J Infect Dis. 2017;215(7):1102-6. https://doi.org/10.1093/infdis/jix031 PMID: 28368456

23. Cowling BJ, Fang VJ, Nishiura $\mathrm{H}$, Chan K-H, Ng S, Ip DKM, et al. Increased risk of noninfluenza respiratory virus infections associated with receipt of inactivated influenza vaccine. Clin Infect Dis. 2012;54(12):1778-83. https://doi.org/10.1093/cid/ cis307 PMID: 22423139

24. Skowronski DM, Zou M, Clarke Q, Chambers C, Dickinson JA, Sabaiduc S, et al. Influenza vaccine does not increase the risk of coronavirus or other noninfluenza respiratory viruses: retrospective analysis from Canada, 2010-2011 to 2016-2017. Clin Infect Dis. 2020;71(16):2285-8. https://doi.org/10.1093/ cid/ciaa626 PMID: 32442261

25. Nickbakhsh S, Ho A, Marques DFP, McMenamin J, Gunson RR, Murcia P. Epidemiology of seasonal coronaviruses: Establishing the context for the emergence of coronavirus disease 2019. J Infect Dis. 2020;222(1):17-25. https://doi. org/10.1093/infdis/jiaa185 PMID: 32296837

26. Hirve S, Crawford N, Palekar R, Zhang W, WHO RSV surveillance Group. Clinical characteristics, predictors, and performance of case definition-Interim results from the WHO global respiratory syncytial virus surveillance pilot. Influenza Other Respir Viruses. 2020;14(6):647-57. https://doi. org/10.1111/irv.12688 PMID: 31670892

27. Williams TC, Kim S, Spiro DJ, Campbell H. Preparing for the future implementation of respiratory syncytial virus vaccines. Lancet Respir Med. 2020;8(3):233-5. https://doi.org/10.1016/ S2213-2600(19)30478-3 PMID: 31978328

28. Jain S, Self WH, Wunderink RG, Fakhran S, Balk R, Bramley AM, et al. Community-acquired pneumonia requiring hospitalization among U.S. adults. N Engl J Med. 2015;373(5):415-27. https:// doi.org/10.1056/NEJMoa1500245 PMID: 26172429

29. Jain S, Williams DJ, Arnold SR, Ampofo K, Bramley AM, Reed C, et al. Community-acquired pneumonia requiring hospitalization among U.S. children. N Engl J Med. 2015;372(9):835-45. https://doi.org/10.1056/NEJMoa1405870 PMID: 25714161

30. Kodama F, Nace DA, Jump RLP. Respiratory syncytial virus and other noninfluenza respiratory viruses in older adults. Infect Dis Clin North Am. 2017;31(4):767-90. https://doi. org/10.1016/j.idc.2017.07.006 PMID: 29079159

31. Pavia AT. Viral infections of the lower respiratory tract: old viruses, new viruses, and the role of diagnosis. Clin Infect Dis. 2011;52(Suppl 4):S284-9. https://doi.org/10.1093/cid/ciro43 PMID: 21460286

32. Schauwvlieghe AFAD, Rijnders BJA, Philips N, Verwijs R, Vanderbeke L, Van Tienen C, et al. Invasive aspergillosis in patients admitted to the intensive care unit with severe influenza: a retrospective cohort study. Lancet Respir Med. 2018;6(10):782-92. https://doi.org/10.1016/S22132600(18)30274-1 PMID: 30076119

33. Blyth CC, Webb SAR, Kok J, Dwyer DE, van Hal SJ, Foo H, et al. The impact of bacterial and viral co-infection in severe influenza. Influenza Other Respir Viruses. 2013;7(2):168-76. https://doi.org/10.1111/j.1750-2659.2012.00360.x PMID: 22487223

34. Teng F, Liu X, Guo S-B, Li Z, Ji W-Q, Zhang F, et al. Communityacquired bacterial co-infection predicts severity and mortality in influenza-associated pneumonia admitted patients. J Infect Chemother. 2019;25(2):129-36. https://doi.org/10.1016/j. jiac.2018.10.014 PMID: 30448361

35. Ieven M, Coenen S, Loens K, Lammens C, Coenjaerts F, Vanderstraeten A, et al. Aetiology of lower respiratory tract infection in adults in primary care: a prospective study in 11 European countries. Clin Microbiol Infect. 2018;24(11):1158-63. https://doi.org/10.1016/j.cmi.2018.02.004 PMID: 29447989

36. Talbot HK, Falsey AR. The diagnosis of viral respiratory disease in older adults. Clin Infect Dis. 2010;50(5):747-51. https://doi. org/10.1086/650486 PMID: 20121411

37. Matsuno O, Kataoka H, Takenaka R, Okubo F, Okamoto $\mathrm{K}$, Masutomo K, et al. Influence of age on symptoms and laboratory findings at presentation in patients with influenzaassociated pneumonia. Arch Gerontol Geriatr. 2009;49(2):3225. https://doi.org/10.1016/j.archger.2008.11.015 PMID: 19150140

\section{License, supplementary material and copyright}

This is an open-access article distributed under the terms of the Creative Commons Attribution (CC BY 4.0) Licence. You may share and adapt the material, but must give appropriate credit to the source, provide a link to the licence and indicate if changes were made.

Any supplementary material referenced in the article can be found in the online version.

This article is copyright of the authors or their affiliated institutions, 2021. 